\title{
Recovery after Three-shift Work: Relation to Sleep-related Cardiac Neuronal Regulation in Nurses
}

\author{
Min-Huey CHUNG', Terry B. J. KUO ${ }^{2,3}$, Nanly $\mathrm{HSU}^{4}$, Hsin $\mathrm{CHU}^{5}$, \\ Kuei-Ru CHOU ${ }^{1}$ and Cheryl C. H. YANG ${ }^{2,3^{*}}$
}

\author{
${ }^{1}$ Graduate Institute of Nursing, College of Nursing, Taipei Medical University, Taipei, Taiwan \\ ${ }^{2}$ Institute of Brain Science, National Yang-Ming University, No. 155, Sec. 2, Linong St., Beitou Dist., Taipei City \\ 11221, Taiwan \\ ${ }^{3}$ Sleep Research Center, National Yang-Ming University, No. 155, Sec. 2, Linong St., Beitou Dist., Taipei City, \\ Taiwan \\ ${ }^{4}$ Department of Nursing, Yuanpei University, No. 306, Yuanpei St., Xiangshan Dist., Hsinchu City, Taiwan \\ ${ }^{5}$ Institute of Aerospace Medicine, School of Medicine, National Defense Medical Center, Taipei, Taiwan
}

\author{
Received May 20, 2011 and accepted November 8, 2011
}

Published online in J-STAGE December 6, 2011

\begin{abstract}
This study was to evaluate whether sleep-related autonomic function in nurses recovers during their days off following a rapidly rotating, clockwise shift schedule. Ten rotating-shift nurses and ten regular morning-shift nurses were included. Nurses slept at home and were allowed to sleep and wake spontaneously. For the rotating-shift workers, ambulatory polysomnographic recordings were taken during nighttime sleep (after the second morning shift, afternoon shift, and on days off) and during daytime sleep (after the second night shift). No significant differences were found between regular-shift nurses and rotating-shift nurses in terms of sleep patterns and cardiac autonomic functions during day shift. When comparing sleep patterns within shift groups, the total sleep time of night shift was lower than their other shifts. Controlling for the variable of total sleep time allowed us to compare cardiac autonomic functions following different shifts (for the rotating shift nurses). During the non-rapid eye movement and rapid eye movement periods, the high frequency (HF) value on rotating shift nurses' days off was found to be significantly higher than their other shifts. However, the low to high frequency ratio (LF/HF) on days off was found to be obviously lower than that during shift work. Two consecutive days off may be sufficient for nurses to recover sleep-related autonomic functions after a rapidly rotating, clockwise three-shift schedule. Sleeprelated autonomic functions may be improved during days off to minimize health risks.
\end{abstract}

Key words: Nurses, Sleep, Autonomic nervous system, Heart rate variability, Shift work

\section{Introduction}

*To whom correspondence should be addressed.

E-mail: cchyang@ym.edu.tw

C2012 National Institute of Occupational Safety and Health
Nurses working rotating three-shifts may work in the morning, afternoon, or night. Working different shifts interrupts their daily schedules significantly, with those working night shifts requiring sleep during the daytime. 
When nurses change shifts, they also change their sleep times, returning to nighttime sleep when they are working morning and afternoon shifts. The resultant disturbed sleep (which is consistently reported more often by shift workers than by day workers) can ultimately lead to reduced alertness and performance ${ }^{1)}$. Furthermore, shift work results in changes to the circadian fluctuations of autonomic activity ${ }^{2}$. Diminished alertness may be ascribed to the reduction in cardiac sympathetic modulation that occurs when people work at night ${ }^{3)}$. In addition, shift workers are known to suffer from relatively higher rates of cardiac, gastrointestinal, and reproductive disorders, which may also be attributable to autonomic imbalance ${ }^{4-6}$.

Analysis of heart rate variability (HRV) could be a useful tool to assess cardiac autonomic control ${ }^{7}$. HRV variables usually differ between working and sleeping time because of differences in autonomic modulation ${ }^{2}$. During sleeping time, delicate alterations in the HRV may be measured, which may not be apparent when the person is awake ${ }^{8}$. Previous studies have indicated that permanent night shift nurses have higher sympathetic nervous activity during nighttime sleep than do regular morning shift nurses ${ }^{9}$. However, few studies have thoroughly monitored sleeping patterns among rotating three-shift nurses, such as measuring changes in their sleep-related cardiac neuronal regulation, or examining whether such patterns can fully recover during the nurses' days off. Parasympathetic activities are related to relaxation ${ }^{10)}$; therefore, the present study used polysomnography (PSG) recordings to examine whether sleep-related cardiac parasympathetic activity is significantly lower during rotating-shift nurses' days off than their other shifts. In terms of cardiac autonomic effects, this result was to help evaluate whether two consecutive days off after working a rotating shift are sufficient to recover for rotating three-shift nurses. Such information would be helpful to consider the health of nurses when scheduling their working shifts.

\section{Methods}

\section{Participants and procedure}

All the participants were female, and all were recruited from one regional hospital in Taipei city, Taiwan. Potential participants were excluded from the study sample if they had any recorded psychopathology, neurological disease, or cardiovascular disease, or if they were using any hypnotics reported to influence sleep patterns, the autonomic nervous system (ANS), or cardiovascular fluctuations. Informed written consent was obtained from all participants, and the experimental protocol was approved by the Ethics Committee of the Tri-Service General Hospital, Taiwan ${ }^{11)}$. A total of 20 nurses were recruited, with ten participants in the control group (those who worked regular morning shifts), and ten participants in the experimental group (those who worked rotating three-shifts).

No significant differences were found between the rotating three-shift nurses and the regular morning-shift nurses for age, body mass index (BMI), number of years employed in nursing, blood pressure, and tea or coffee consumption (Table 1). In the regular morning-shift group, nurses worked five consecutive morning shifts (from 0800 to 1600) and had two days off per week. They had been employed as nurses for approximately three years. In the rotating three-shift group, nurses worked on a fast-forward rotating shift schedule with the following sequence: three consecutive morning shifts ( 0730 to 1530 ), followed by three afternoon shifts (1530 to 2330), followed by three night shifts (2330 to 0730 ); the nurses had two or three days off between the cycles. This group had been working as nurses for approximately two years. Nurses were allowed a day of habituation after their last working shift. Thereafter, polysomnographic recordings were conducted during nighttime sleep (after the second morning shift, afternoon shift, and days off) and during daytime sleep (after the second night shift).

\section{Data recording}

For the sleep-stage analysis, data files were converted into European Data Format and imported into commercial sleep analysis software (Somnologica 3.1.2, Embla, U.S.A.). The computer-assisted sleep analysis was conducted according to the criteria defined by Rechtschaffen and Kales $^{12)}$. The results were verified by a qualified sleep technician.

Recordings were made by electroencephalography (EEG, C3/Cz), electro-oculography (EOG), sub-mental electromyography (EMG), and electrocardiography (ECG). The readings were synchronously digitized and stored on a memory card throughout the day or night using an ambulatory recorder. Signals were amplified with different multipliers for the filter bandwidths, in a similar manner to that reported in a previous study on data acquisition systems, as follows: EEG (5000), EMG (2000), ECG (5000), and EOG $(1000)^{13)}$. The EEG and EMG signals were both filtered at 0.32 to $40 \mathrm{~Hz}$, whereas the EOG signal was filtered at 0.032 to $40 \mathrm{~Hz}$, and the ECG signal was filtered at 0.64 to $40 \mathrm{~Hz}$. These bioelectric signals were relayed to an eight-bit analog-digital converter connected to 
an IBM PC-compatible computer. Although the signals were synchronously digitized, they were measured at different sampling rates of $128 \mathrm{~Hz}$ for the EEG, EOG, and EMG signals, and $256 \mathrm{~Hz}$ for the ECG signal. The acquired data were analyzed online and simultaneously stored on a hard disk for subsequent offline verification.

\section{Digital signal processing}

The ECG signals were preprocessed according to recommended procedures ${ }^{14)}$, which were fully described in our previous studies ${ }^{15)}$. Briefly, the computer algorithm identified each normal ventricular discharge waveform and rejected any ventricular premature complex, or noise, according to its likelihood in a standard template. Stationary R-R intervals (RR) were resampled and interpolated at a rate of $8 \mathrm{~Hz}$ to provide continuity in the time domain. The sampling rate of the EEG signals was reduced to $64 \mathrm{~Hz}$.

\section{Power spectral analysis}

The analyzed EEG, EOG, and RR signals were truncated into successive 64-second time segments (windows) with a 50\% (32 s) overlap. A Hamming window was applied to each time segment to attenuate the leakage effect ${ }^{13)}$. Our algorithm then estimated the power density of the spectral components, based on a fast Fourier transformation ${ }^{16)}$. The resultant power spectrum was corrected for attenuation stemming from sampling and application of the Hamming window $^{13,15)}$.

The analyzed EMG signals, which were truncated into successive 16-second time segments with no overlap, were consequently subjected to fast Fourier transformation after applying the Hamming window. Four successive EMG spectra (covering a total of $64 \mathrm{~s}$ ) were averaged to achieve synchronization with the EEG and RR spectra. For each 64-s time segment, we quantified the high-frequency (HF) ( 0.15 to $0.4 \mathrm{~Hz})$ and low-frequency (LF) (0.04 to 0.15 $\mathrm{Hz})$ power of the RR spectrogram ${ }^{16)}$, and the delta power ( 0.5 to $4 \mathrm{~Hz}$ ) of the EEG spectrogram. The HF is usually interpreted as an index of vagal modulation, whereas the LF is computed as measuring a mixture of sympathetic modulation and parasympathetic modulation. The LF/HF ratio mainly reflects the balance of the ANS, with higher values implying either greater sympathetic or less vagal influence ${ }^{14)}$.

\section{Statistical analysis}

To ensure validity of the comparisons between the control (morning shift) and experimental (rotating shift) groups, the first step of the analysis compared the basic characteristics of the participants. Demographic variables, years of employment, blood pressure, and tea/coffee consumption were presented as the mean \pm standard deviation (SD) for continuous variables, and as discrete numbers for categorical variables. Statistical comparisons were made using $\chi^{2}$ tests.

The next step of the analysis involved more complex and specific calculations. Two comparison scenarios were included in the study design: 1) a comparison between the nighttime sleep of rotating three-shift nurses after their morning shifts, and the nighttime sleep of regular morningshift nurses; we used the Mann-Whitney U-tests; and 2) comparisons of rotating-shift nurses on the nighttime sleep of morning shifts, afternoon shifts, days off and daytime sleep of night shifts; we used Friedman's test, which was also used to analyze the data that did not fit the assumptions of parametric parameters ${ }^{17)}$.

Generalized Estimating Equation (GEE) models were first introduced by Zeger and Liang in $1986^{18)}$. These are generalized linear models that can be used with either normal or non-normal distributions; they can also capture the correlations within participants and between groups to offer efficient estimates for repeat measurements. In this study, we performed GEE with an identity link and normal distribution to test the primary study hypothesis; that is, to investigate the association between cardiac autonomic function and shift pattern by controlling the covariate of total sleep time. In addition, GEE enables us to conduct multiple comparisons across different shift work categories using the Bonferroni post hoc procedures. All statistical analyses were performed using SPSS version 17.0 for Windows (SPSS Inc., Chicago, IL, U.S.A.). Probability values of $p<0.05$ were considered statistically significant.

\section{Results}

In the baseline comparisons, no significant difference was identified between the two groups (Table 1). Regarding sleeping patterns (Table 2) and cardiac autonomic functions (Table 3), no significant differences emerged for the nighttime sleep of nurses working regular morning shifts and those working mornings on a rotating three-shift schedule. Regarding total sleep time (Table 2), a significant difference was found within the experimental group. Therefore, the comparison of cardiac autonomic function was achieved by controlling total sleep time within the experimental group, as shown in Table 3. During the nonrapid eye movement (NREM) and rapid eye movement 
Table 1. Basic characteristics of participants

\begin{tabular}{lccc}
\hline & \multicolumn{2}{c}{ Group } & \\
\cline { 2 - 3 } Variable & $\begin{array}{c}\text { Control group } \\
(n=10)\end{array}$ & $\begin{array}{c}\text { Experimental group } \\
(n=10)\end{array}$ & $p$ value $^{\wedge}$ \\
\hline Age (yr) & $29.0 \pm 2.9$ & $27.1 \pm 1.9$ & 0.14 \\
Years employed in nursing & $3.0 \pm 2.1$ & $2.1 \pm 0.9$ & 0.56 \\
Body mass index & $20.0 \pm 1.1$ & $19.9 \pm 1.9$ & 0.45 \\
Systolic blood pressure (mmHg) & $117.6 \pm 6.4$ & $112.8 \pm 4.4$ & 0.07 \\
Diastolic blood pressure (mmHg) & $71.4 \pm 5.2$ & $67.4 \pm 4.0$ & 0.07 \\
Tea consumption & & & \\
Never & 4 & 1 & 0.32 \\
$<1$ time per week & 1 & 4 & \\
$1-2$ times per week & 3 & 1 & \\
3-4 times per week & 1 & 0 & \\
5-7 times per week & 1 & & \\
Coffee consumption & & 4 & \\
Never & 5 & 2 & \\
$<1$ time per week & 1 & 2 & \\
1-2 times per week & 2 & 1 & \\
3-4 times per week & 0 & & \\
5-7 times per week & 2 & & \\
\hline
\end{tabular}

$\widehat{\wedge}$ Mann-Whitney $U$ tests were used for continuous variables and $\chi^{2}$ tests for categorical variables.

Control group (regular morning shift nurses); Experimental group (rotating three-shift work nurses).

Table 2. Differences in sleep patterns among nurses working different shifts

\begin{tabular}{|c|c|c|c|c|c|c|}
\hline \multirow[b]{2}{*}{ Variable } & \multirow{2}{*}{$\begin{array}{l}\text { Control group } \\
\qquad(n=10)\end{array}$} & \multicolumn{5}{|c|}{ Experimental group $(n=10)$} \\
\hline & & $\begin{array}{l}\text { Morning shift; } \\
\text { sleep at night }\end{array}$ & $\begin{array}{l}\text { Afternoon shift; } \\
\text { sleep at night }\end{array}$ & $\begin{array}{c}\text { Night shift; } \\
\text { sleep in the day }\end{array}$ & $\begin{array}{c}\text { Days off; } \\
\text { sleep at night }\end{array}$ & $p$ value $^{\wedge}$ \\
\hline Total sleep time (min) & $359.9 \pm 43.4$ & $348.4 \pm 90.9$ & $377.8 \pm 90.7$ & $274.20 \pm 72.0$ & $377.8 \pm 104.5$ & $p<0.05$ \\
\hline Wake after sleep onset (min) & $31.5 \pm 28.6$ & $23.2 \pm 30.9$ & $18.4 \pm 8.5$ & $14.88 \pm 7.1$ & $25.2 \pm 17.7$ & 0.80 \\
\hline Sleep onset latency (min) & $15.5 \pm 12.9$ & $27.9 \pm 36.2$ & $19.6 \pm 28.3$ & $6.35 \pm 4.6$ & $11.7 \pm 12.9$ & 0.47 \\
\hline Sleep efficiency, $\%$ & $89.1 \pm 8.2$ & $86.6 \pm 13.0$ & $91.2 \pm 6.0$ & $92.7 \pm 2.9$ & $91.6 \pm 3.5$ & 0.99 \\
\hline NREM (\%) & $76.3 \pm 6.9$ & $72.9 \pm 4.3$ & $75.2 \pm 5.6$ & $74.1 \pm 5.6$ & $74.7 \pm 3.7$ & 0.39 \\
\hline REM (\%) & $23.7 \pm 6.9$ & $27.1 \pm 4.3$ & $24.6 \pm 4.6$ & $25.9 \pm 5.6$ & $25.3 \pm 3.7$ & 0.39 \\
\hline Awakening index (times/h) & $4.0 \pm 1.5$ & $3.4 \pm 0.9$ & $3.3 \pm 0.9$ & $3.4 \pm 1.4$ & $4.0 \pm 1.1$ & 0.52 \\
\hline Arousal index (times/h) & $3.9 \pm 1.6$ & $3.1 \pm 1.4$ & $3.0 \pm 0.9$ & $2.8 \pm 1.3$ & $3.1 \pm 1.2$ & 0.46 \\
\hline
\end{tabular}

1. ${ }^{\wedge}$ The sleep patterns were compared within the experimental group using the Friedman's test.

2. Control group (regular morning-shift nurses); Experimental group (rotating three-shift work nurses); NREM, non-rapid eye movement sleep; REM, rapid eye movement sleep.

(REM) periods, the HF power on days off was found to be significantly higher than their other shifts in the experimental group. However, the LF/HF value of days off was found to be significantly lower than their other shifts.

\section{Discussion}

This study found that nurses working rotating three- shift schedules displayed higher parasympathetic activity during their nighttime sleep after two consecutive days off, compared to their nighttime sleep during morning shift work. Our study addressed whether nighttime sleep after two consecutive days off can counteract the deleterious effects of the three-shift rotation system. Comparing the nighttime sleep of nurses working morning shifts on a rotating threeshift basis with the nighttime sleep of regular morning-shift 
Table 3. Comparisons of cardiac autonomic function among nurses working different shifts

\begin{tabular}{|c|c|c|c|c|c|c|c|}
\hline \multirow[b]{2}{*}{ Variable } & \multirow{2}{*}{$\begin{array}{l}\text { Control group } \\
\qquad(n=10)\end{array}$} & \multicolumn{6}{|c|}{ Experimental group $(n=10)$} \\
\hline & & $\begin{array}{l}\text { Morning shift; } \\
\text { sleep at night(A) }\end{array}$ & $\begin{array}{l}\text { Afternoon shift; } \\
\text { sleep at night(B) }\end{array}$ & $\begin{array}{c}\text { Night shift; } \\
\text { sleep in day(C) }\end{array}$ & $\begin{array}{c}\text { Days off; } \\
\text { sleep at night(D) }\end{array}$ & $p$ value ${ }^{\wedge}$ & $\begin{array}{c}\text { Multiple } \\
\text { comparison }\end{array}$ \\
\hline \multicolumn{8}{|l|}{ NREM sleep } \\
\hline $\mathrm{RR}(\mathrm{ms})$ & $914.99 \pm 52.42$ & $931.93 \pm 68.80$ & $952.00 \pm 71.90$ & $904.03 \pm 91.36$ & $972.05 \pm 82.43$ & $p<0.001$ & $\mathrm{C}<\mathrm{A}<\mathrm{B}<\mathrm{D}$ \\
\hline $\mathrm{HF}\left[\ln \left(\mathrm{ms}^{2}\right)\right]$ & $6.22 \pm 0.66$ & $6.24 \pm 0.60$ & $6.22 \pm 0.57$ & $5.98 \pm 0.72$ & $6.65 \pm 0.50$ & $p<0.001$ & $\mathrm{C}<\mathrm{B}<\mathrm{A}<\mathrm{D}$ \\
\hline $\operatorname{LF}\left[\ln \left(\mathrm{ms}^{2}\right)\right]$ & $6.18 \pm 0.58$ & $6.16 \pm 0.41$ & $6.35 \pm 0.39$ & $6.06 \pm 0.59$ & $6.52 \pm 0.39$ & $p<0.001$ & $(\mathrm{C}, \mathrm{A})<\mathrm{B}<\mathrm{D}$ \\
\hline LF:HF (ratio) & $-0.038 \pm 0.40$ & $-0.11 \pm 0.43$ & $0.13 \pm 0.42$ & $0.18 \pm 0.54$ & $-0.15 \pm 0.40$ & $p<0.05$ & $\mathrm{D}<\mathrm{A}<(\mathrm{B}, \mathrm{C})$ \\
\hline \multicolumn{8}{|l|}{ REM sleep } \\
\hline $\mathrm{RR}(\mathrm{ms})$ & $882.89 \pm 43.15$ & $902.20 \pm 53.58$ & $923.53 \pm 82.28$ & $886.54 \pm 78.69$ & $938.80 \pm 79.54$ & $p<0.001$ & $\mathrm{C}<\mathrm{A}<\mathrm{B}<\mathrm{D}$ \\
\hline $\mathrm{HF}\left[\ln \left(\mathrm{ms}^{2}\right)\right]$ & $5.91 \pm 0.56$ & $6.08 \pm 0.54$ & $6.09 \pm 0.65$ & $5.74 \pm 0.77$ & $6.31 \pm 0.48$ & $p<0.001$ & $\mathrm{C}<(\mathrm{A}, \mathrm{B})<\mathrm{D}$ \\
\hline $\mathrm{LF}\left[\ln \left(\mathrm{ms}^{2}\right)\right]$ & $6.71 \pm 0.44$ & $6.87 \pm 0.45$ & $6.86 \pm 0.58$ & $6.43 \pm 0.69$ & $6.86 \pm 0.49$ & $p<0.05$ & $\mathrm{C}<(\mathrm{A}, \mathrm{B}, \mathrm{D})$ \\
\hline LF:HF (ratio) & $0.78 \pm 0.35$ & $0.75 \pm 0.42$ & $0.78 \pm 0.44$ & $0.79 \pm 0.46$ & $0.65 \pm 0.45$ & $p<0.001$ & $\mathrm{D}<(\mathrm{A}, \mathrm{B}, \mathrm{C})$ \\
\hline
\end{tabular}

nurses, we found that both groups achieved similar patterns and cardiac neuronal modulation during sleep (Tables 2 and 3). We also examined the sleep-related cardiac neuronal regulation of the nurses' nighttime sleep after working morning shifts, and found that their daytime sleep while working night shifts evidenced higher sympathetic activity and lower parasympathetic activity. However, during their nighttime sleep on days off, the parasympathetic function reflected by HF was enhanced and the sympathetic function indicated by LF/HF was diminished, which readjusted the sleep-related cardiac neuronal regulation (Table 3). Thus, the current study indicated that at least two consecutive days off may be sufficient for rotating three-shift nurses to restore their nighttime sleep to the same quality as that of dayshift nurses.

Previous studies have reported that sleepiness increases when nurses work night shifts ${ }^{19,}$; ; however, few studies have compared the sleep-related ANS functions associated with different shift patterns among rotating three-shift nurses. The measurements of autonomic nervous function during waking hours might not reflect the condition of the ANS during sleep ${ }^{8,21)}$. A number of delicate alterations, such as sympathetic activation accompanied by shift work, cannot be detected easily if a sleep study is not performed ${ }^{9)}$. This study revealed that slight but important differences in cardiac autonomic regulation occurred during sleep between shifts.
Shift workers are more vulnerable than day workers to adverse health effects ${ }^{22)}$; therefore, questions on how shift schedules can be improved to minimize health risks deserve considerable attention. The current study showed that the distribution of free time between shifts can improve sleep-related cardiac autonomic regulation (Table 3). This result was inconsistent with that of a previous study, which indicated that several coronary risk factors worsened after a week off compared to measurements at the end of a shift sequence ${ }^{22)}$. Differences in health states between days off and a week off may be attributable to the distribution of free time and activities outside the workplace. In the current study, all morning shifts were preceded by afternoon or night and shifts with days off in between the shifts. Nurses are usually required to work five days a week for eight-hour shifts; therefore, they have a tendency to stay at home to take a rest during their days off. This circumstance explains how the sleep-related cardiac autonomic function can rebound to counteract the detrimental effect of the three-shift rotation on nurses.

ANS during NREM and REM sleep are known to have different changes ${ }^{9,16)}$; therefore, the changes of ANS distinguished according to NREM and REM sleep would be more correct. This study indicated the value of HF was higher during NREM sleep than during REM sleep. In contrast, the value of $\mathrm{LF} / \mathrm{HF}$ was higher during REM sleep than during 
NREM sleep (Table 3). Sleep characterized by autonomic nervous activity was in line with the findings of prior studies, which revealed a sympathetic dominance in the autonomic system during REM sleep and the major influence of vagal activity during NREM sleep ${ }^{23,24)}$. In addition, if the LF/HF was higher in NREM sleep, this phenomenon was associated with more-frequent interruptions during sleep ${ }^{25}$. Thus our results for the autonomic regulation were presented for NREM sleep and REM sleep separately.

The present study found the HF and LF values on days off was significantly higher than in the daytime sleep after night work (Table 3 ). The results obtained with HRV variables showed that parasympathetic activity was decreased after night shifts and rebounded during days off. In other words, the decreased parasympathetic activity may show that the recovery after work in nurses was poorer as reflected by lower HF and LF value ${ }^{26}$. As a result, the higher HF value indicated that cardiac vagal activity was higher during nurses' days off than their other shifts in the rotating three-shift nurses. This finding supports the notion that distribution of free time is an important factor for maintaining health when working a rapidly rotating, clockwise shift schedule.

Numerous factors may affect the sleep-related cardiac autonomic system, such as age ${ }^{15)}, \mathrm{BMI}^{27)}$, blood pressure ${ }^{28)}$, tea/coffee consumption ${ }^{29)}$, and sleep patterns ${ }^{25)}$. In the present sample, no differences were present between the control and experimental groups regarding age, BMI, the number of years employed in nursing, blood pressure, and tea/coffee consumption. However, in the aspect of sleep patterns, night-shift workers tended to exhibit shorter total sleep time than morning-shift workers. The results of the current study were consistent with those of prior research, in that daytime sleep after night work was found to be of shorter duration than nighttime sleep ${ }^{30,31)}$. Although nurses usually feel exhausted after a night shift, the daytime circadian rhythm interferes with sleep; thus, night shift nurses may experience less need for sleep.

Interpretation of the present results was affected by several conditions. First, our sample of nurses was small, which meant that it had low statistical power, particularly for finding differences among shifts. However, our results indicated that nurses must distribute their free time appropriately. Second, daily activities were not monitored during days off. Large variations of daily activities could contribute to changes in cardiovascular autonomic functions. Our findings showed that shift work nurses endured augmented sympathetic activity and attenuated vagal activity when working afternoon and night shifts, compared to on their days off (Table 3). That is, during their free time between shifts, their physiological functioning appeared to revert to normal; this suggests that nurses' days off are sufficient to allow them to recover and improve their sleep-related cardiovascular regulation. Third, this research was a cross-sectional study; therefore, the analysis of variables and inferences drawn from the analysis were limited to a particular point in time. Finally, possible causal relationships between the nurses' free time and sleep-related cardiovascular regulation require further exploration.

\section{Conclusions}

For nurses in the rotating-shift group, sleep-related cardiac neuronal regulation improved during their days off. This finding supports that the distribution of free time is an important factor for nurses' health, and indicates that nurses should plan their free time between shifts carefully, especially if they are working a rapidly rotating, clockwise schedule. Rotating three-shift work may be shown to be acceptable if nurses distribute their free time appropriately. Another implication of these finding is the duration of days off. Our study found that the nighttime sleep of two consecutive days off was related to increased parasympathetic activity after afternoon and night shifts. However, other types of shift systems, and recovery of autonomic modulation after different numbers of consecutive days off, were not compared in the present study. These aspects could be further examined in studies using larger samples.

\section{References}

1) Åkerstedt T (2003) Shift work and disturbed sleep/ wakefulness. Occup Med (Lond) 53, 89-94.

2) Ito H, Nozaki M, Maruyama T, Kaji Y, Tsuda $Y$ (2001) Shift work modifies the circadian patterns of heart rate variability in nurses. Int J Cardiol 79, 231-6.

3) Furlan R, Barbic F, Piazza S, Tinelli M, Seghizzi P, Malliani A (2000) Modifications of cardiac autonomic profile associated with a shift schedule of work. Circulation 102, 1912-6.

4) Callister R, Suwarno NO, Seals DR (1992) Sympathetic activity is influenced by task difficulty and stress perception during mental challenge in humans. J Physiol 454, 373-87.

5) Muecke S (2005) Effects of rotating night shifts: literature review. J Adv Nurs 50, 433-9.

6) Wilson JL (2002) The impact of shift patterns on healthcare professionals. J Nurs Manag 10, 211-9.

7) Van Amelsvoort LG, Schouten EG, Maan AC, Swenne CA, 
Kok FJ (2000) Occupational determinants of heart rate variability. Int Arch Occup Environ Health 73, 255-62.

8) Kuo TBJ, Lai CJ, Shaw FZ, Lai CW, Yang CCH (2004) Sleep-related sympathovagal imbalance in SHR. Am J Physiol Heart Circ Physiol 286, H1170-6.

9) Chung MH, Kuo TBJ, Hsu N, Chu H, Chou KR, Yang CCH (2009) Sleep and autonomic nervous system changes enhanced cardiac sympathetic modulations during sleep in permanent night shift nurses. Scand J Work Environ Health 35, 180-7.

10) Chuang CY, Han WR, Li PC, Young ST (2010) Effects of music therapy on subjective sensations and heart rate variability in treated cancer survivors: a pilot study. Complement Ther Med 18, 224-6.

11) Touitou Y, Smolensky MH, Portaluppi F (2006) Ethics, standards, and procedures of animal and human chronobiology research. Chronobiol Int 23, 1083-96.

12) Rechtschaffen A, Kales A (1968) A manual of standardized terminology, techniques, and scoring system for sleep stages of Human subjects. Psychopharmacology (Berl) 181, 270-9.

13) Kuo TBJ, Chan SH (1993) Continuous, on-line, real-time spectral analysis of systemic arterial pressure signals. Am J Physiol 264, H2208-13.

14) Task Force of the European Society of Cardiology and the North American Society of Pacing and Electrophysiology (1996) Heart rate variability: standards of measurement, physiological interpretation and clinical use. Circulation 93, 1043-65.

15) Kuo TBJ, Lin T, Yang CCH, Li CL, Chen CF, Chou P (1999) Effect of aging on gender differences in neural control of heart rate. Am J Physiol 277, H2233-9.

16) Yang CCH, Lai CW, Lai HY, Kuo TBJ (2002) Relationship between electroencephalogram slow-wave magnitude and heart rate variability during sleep in humans. Neurosci Lett 329, 213-6.

17) Hatch E, Lazaraton A (1991) The research manual: design and statistics for applied linguistics. Heinle \& Heinle Pub, Boston.

18) Zeger SL, Liang KY (1986) Longitudinal data analysis for discrete and continuous outcomes. Biometrics 42, 121-30.

19) Åkerstedt T, Kecklund G, Knutsson A (1991) Manifest sleepiness and the spectral content of the EEG during shift work. Sleep 14, 221-5.
20) Kecklund G, Åkerstedt T (1995) Effects of timing of shifts on sleepiness and sleep duration. J Sleep Res 4, 47-50.

21) Baharav A, Kotagal S, Gibbons V, Rubin BK, Pratt G, Karin J, Akselrod S (1995) Fluctuations in autonomic nervous activity during sleep displayed by power spectrum analysis of heart rate variability. Neurology 45, 1183-7.

22) Axelsson J, Lowden A, Kecklund G (2006) Recovery after shift work: relation to coronary risk factors in women. Chronobiol Int 23, 1115-24.

23) Endo S, Kobayashi T, Yamamoto T, Fukuda H, Sasaki M, Ohta T (1981) Persistence of the circadian rhythm of REM sleep: a variety of experimental manipulations of the sleepwake cycle. Sleep 4, 319-28.

24) Mancia G (1993) Autonomic modulation of the cardiovascular system during sleep. N Engl J Med 328, 347-9.

25) Kuo TBJ, Shaw FZ, Lai CJ, Lai CW, Yang CCH (2004) Changes in sleep patterns in spontaneously hypertensive rats. Sleep 27, 406-12.

26) Martinmäki K, Rusko H, Kooistra L, Kettunen J, Saalasti S (2006) Intraindividual validation of heart rate variability indexes to measure vagal effects on hearts. American Journal of Physiology - Heart and Circulatory Physiology 290, H640-7.

27) Schmid K, Schonlebe J, Drexler H, Mueck-Weymann M (2010) Associations between being overweight, variability in heart rate, and well-being in the young men. Cardiol Young 20, 54-9.

28) Chao AC, Chern CM, Kuo TB, Chou CH, Chuang YM, Wong WJ, Hu HH (2003) Noninvasive assessment of spontaneous baroreflex sensitivity and heart rate variability in patients with carotid stenosis. Cerebrovasc Dis 16, $151-7$.

29) Barry RJ, Clarke AR, Johnstone SJ, Rushby JA (2008) Timing of caffeine's impact on autonomic and central nervous system measures: clarification of arousal effects. Biol Psychol 77, 304-16.

30) Cao CG, Weinger MB, Slagle J, Zhou C, Ou J, Gillin S, Sheh B, Mazzei W (2008) Differences in day and night shift clinical performance in anesthesiology. Hum Factors 50, 276-90.

31) Isah EC, Iyamu OA, Imoudu GO (2008) Health effects of night shift duty on nurses in a university teaching hospital in Benin City, Nigeria. Niger J Clin Pract 11, 144-8. 